\title{
Tratamento alternativo na síndrome da angústia respiratória, no adulto: relato de caso
}

Valéria R. COSTA*, Ana Maria P. R. SILVA*, Vera Regina M. COIMBRA*, Maria Ignês FELTRIN*, Fábio B. JATENE*, Marcelo B. M. AMATO*, José Otávio C. AULER JÚNIOR*

RBCCV $44205-133$

COSTA, V. R.; SILVA, A. M. P.; COIMBRA, V. R. M.; FELTRIM, M. I.; JATENE, F. B.; AMATO, M. B. M.; AULER JÚNIOR, J. O. C. - Tratamento alternativo na síndrome da angústia respiratória, no adulto. Rev. Bras. Cir. Cardiovasc., 6(1): 54-58, 1991.

RESUMO: Paciente do sexo feminino, de 33 anos de idade, submetida a revascularização do miocárdio, aneurismectomia e trombectomia de ventrículo esquerdo, evoluiu no pós-operatório com síndrome da angústia respiratória do adulto (SARA). Inicialmente, recebeu tratamento convencional e, pela hipoxemia persistente, optou-se pela remoçâo de $\mathrm{CO}_{2}$ através de oxigenador de membrana (MACCHI-ECCO $\mathrm{R}$ ), associado a pressão positiva de baixa frequeência, com sucesso. A paciente permaneceu sob assistência ventilatória por 57 dias, sendo sete dias com $\mathrm{ECCO}_{2} \mathrm{R}$. Teve alta hospitalar no 83: dia de pós-operatório. Após quatro meses, apresentava, à radiogafia pulmonar, tônus e trofismo muscular normais e espirometria com obstrução em pequenas vias aéreas grau I.

DESCRITORES: síndrome da angústia respiratória, cirurgia, adultos.

\section{INTRODUÇÃO}

A síndrome da angústia respiratória do adulto (SARA) caracteriza-se por insuficiência respiratória aguda e progressiva conseqüente a importante diminuição da complacência pulmonar, necessitando, com freqüência, de assistência ventilatória prolongada. É uma das complicaçōes presentes no pós-operatório de cirurgia cardiaca, com alta taxa de morbidade e mortalidade.

$\mathrm{Na}$ busca de um tratamento efetivo para a SARA. GATTINONE et alii ${ }^{3}$ desenvolveram, em 1979, a técnica de remoção extracorpórea do $\mathrm{CO}_{2}$ através de oxigenador de membrana $\left(\mathrm{ECCO}_{2} \mathrm{R}\right)$.

Esta técnica compreende as duas principais funções pulmonares: remoção do $\mathrm{CO}_{2}$ e transporte do oxigênio. A primeira é realizada por intermédio de um pulmāo de membrana extracorpórea através de bypass veia-veia com fluxo lento, preservando, assim, o fluxo sangüíneo pulmonar. A segunda função é obtida pela difusão do oxigênio no próprio pulmão doente, que é ventilado duas a três vezes por minuto, para manter a mecânica e os volumes pulmonares (Figura 1).

Neste trabalho relatamos o caso de paciente submetida a cirurgia cardíaca, que apresentou SARA no pós-operatório e foi tratada com sucesso por esse método.

\section{RELATO DO CASO}

Paciente do sexo feminino, 33 anos, $56 \mathrm{~kg}$, com diagnóstico de insuficiência coronária e aneurisma de ventrículo esquerdo, com antecedentes de hipertensāo arterial sistêmica (HAS), embolia de membro inferior di-

Trabalho realizado no Instituto do Coraçāo do Hospital das Clinicas da Faculdade de Medicina da Universidade de São Paulo. São Paulo, SP. Brasil.

Recebido para publicação em 20 de março de 1991.

* Do Instituto do Coraçăo do Hospital das Clinicas da Faculdade de Medicina da Universidade de Săo Paulo.

Endereço para separatas: Valéria R. Costa. Av. Dr. Enéas Carvalho de Aguiar, 44. Divisão de Anestesia. 05403 Săo Paulo, SP, Brasil. 
COSTA, V. R.; SILVA, A. M. P.; COIMBRA, V. R. M.; FELTRIM, M. I.; JATENE, F. B.; AMATO, M. B. M.; AULER JÚNIOR, J. O. C. - Tratamento alternativo na sindrome da angústia respiratória, no adulto. Rev. Bras. Cir. Cardiovasc., 6(1): 54-58, 1991.

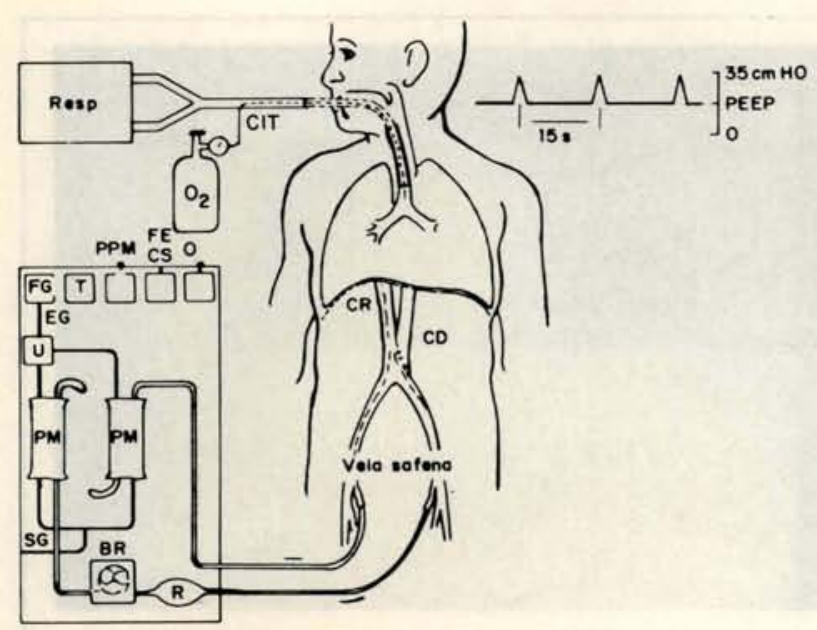

Fig. 1-Acessos e circuito de perfusão para a remoção extracorpórea de $\mathrm{CO}_{2} \cdot \mathrm{CD}=$ cateter de drenagem do sangue; $\mathrm{FECS}=$ fluxo extracorpóreo de sangue; $F G=$ monitor de fluxo de gases: $E G$ = entrada de gases; $S G=$ saida de gases; $U$ = umidificador; $\mathrm{CTT}$ = cateter intratraqueal; $\mathrm{PM}=$ pulmão de membrana; $\mathrm{O}_{2} \%=$ monitor de oxigênio do sangue venoso drenado: PPM = pressâo do pulmăo de membrana, entrada e saida; $\mathrm{R}=$ reservatório venoso; $\mathrm{CR}=$ cateter de retorno venoso do sangue; Resp = respirador; $B R=$ bomba de rolete; PEEP = pressão positiva expiratória final; $T=$ controle da temperatura ambiente ( de Gattinoni Pesenti, Mascheroni, et alii: JAMA, 256: 881, 1986 (com permissão).

reito e tabagismo. Infarto agudo do miocárdio (IAM) há um ano, submetida a revascularizaçāo do miocárdio (RM) com implante de ponte de safena para descendente anterior, mamária esquerda para diagonal e mamária direita para diagonalis, aneurismectomia e trombectomia do ventrículo esquerdo.

Na sala cirúrgica, apresentou hipotensāo arterial pós circulação extracorpórea (CEC), sendo monitorizada com cateter de Swan-Ganz. A proteção miocárdica foi realizada através do gelo amorfo depositado dentro do saco pericárdico e solução cardioplégica.

No pós-operatório (PO) imediato, a paciente evoluiu em baixo débito cardiaco, taquicardia, sangramento por vias aéreas superiores e a extubação procedeu-se em 15 horas. Após extubação, apresentou acentuação do quadro de insuficiência respiratória, associado a diminuição da expansibilidade torácica, taquipnéia, movimentaçāo paradoxal toracoabdominal, oligúria e, à radiografia torácica, velamento das bases (Figura 2A). Foi reintubada no 4 : PO, retornando à assistência ventilatória com uso de pressão positiva expiratória final (PEEP). Com o prolongamento do quadro de insuficiência respiratória, optou-se pela taqueostomia no 8 : PO

No 11: PO, apresentou bradicardia, hipotensão e acentuação do quadro respiratório com diminuição significativa da complacência pulmonar e grave a hipoxemia, caracterizando o quadro clínico de SARA. Como as medidas convencionais de suporte ventilatório nāo melho- raram o quadro, optou-se, no 12 : $\mathrm{PO}$, pelo tratamento através da $\mathrm{ECCO}_{2} \mathrm{R}$, obedecendo-se ao protocolo estabelecido, onde se mantinha sedação e curarização, assistência ventilatória mecânica na modalidade controlada com parâmetros de freqüência respiratória $(f)$ de 4 a $10 \mathrm{rpm}$, fração inspirada de oxigênio $\left(\mathrm{FiO}_{2}\right)$ de 0,7 a 0,9 , pressão positiva expiratória final (PEEP) de 18 a $28 \mathrm{cmH}_{2} \mathrm{O}$ e volume corrente (VC) de 250 a $350 \mathrm{ml}$ com objetivo de manter saturação arterial de oxigênio $\left(\mathrm{SatO}_{2}\right)$ entre 90 e $95 \%$ e niveis de $\mathrm{CO}_{2}$ exalado de 40 a $45 \mathrm{mmHg}$. Foi oferecido suporte nutricional rico em gorduras e pobre em hidrato de carbono e estabelecido controle de temperatura corpórea rígido para diminuir a produção de $\mathrm{CO}_{2}$. Durante esse período, que decorreu do $12^{\circ}$ ao $20^{\circ}$. PO, a paciente apresentou insuficiência renal, necessitando de ultrafiltração e hemodiálise. Isolou-se, na cultura de secreção traqueal, Stafilococcus aureus. Após a retirada da $\mathrm{ECCO}_{2} \mathrm{R}$, retirou-se a curarização e diminuiu-se, gradativamente, a sedação, reintroduzindo-se, progressivamente, a assistência ventilatória mecânica, à modalidade assistida-controlada, mantendo-se PEEP elevado (entre 14 e $15 \mathrm{cmH}_{2} \mathrm{O}$ ) e VC entre 250 a $300 \mathrm{ml}$, com objetivo de manter pico de pressão inspiratório máximo (PPI) de $30 \mathrm{cmH}_{2} \mathrm{O}$. Esse período compreendeu entre o 21: e 29: $\mathrm{PO}$, sendo que a fisioterapia ortopédica teve início no 23 : PO, com exercícios de membros, a fim de melhorar o trofismo e o tônus muscular e preservar a amplitude articular, progredindo para exercícios ativos.

Com a melhora clínica, iniciou-se o desmame do ventilador na modalidade ventilação mandatória intermitente sincronizada (SIMV), com uso de pressão suporte (PS), partindo dos seguintes valores: $f=4 \mathrm{rpm}, \mathrm{F}, \mathrm{O}_{2}$ $=0,4, \mathrm{PEEP}=\mathrm{cmJ}_{2} \mathrm{O}, \mathrm{VC}=350 \mathrm{ml}$, PS $=\mathrm{cmH}_{2} \mathrm{O}$, mantendo $\mathrm{PPI} \leqslant 40 \mathrm{cmH}_{2} \mathrm{O}$. O desmame obedeceu ao protocolo, onde, primeiramente, diminuiu-se o PEEP até $05 \mathrm{cmH}_{2} \mathrm{O}$; a seguir, diminuiu-se a f até $01 \mathrm{rpm} \mathrm{e}$, finalmente, diminuiçāo da PS. Quando seria iniciado o desmame da PS, a paciente apresentou taquipnéia, queda do volume corrente exalado, desconforto respiratório e piora radiológica (Figura $2 \mathrm{~B}$ ), necessitando de aumento do valor de PEEP para $10 \mathrm{cmH}_{2} \mathrm{O}$ e da PS para 25 $\mathrm{CmH}_{2} \mathrm{O}$. Através da ultrassonografia abdominal, para visibilização das cúpulas diafragmáticas, observou-se movimentação minima das mesmas. A fisioterapia enfatizou a estimulação diafragmática, através de exercícios respiratórios na posição sentada reclinada para a frente.

No 49: PO, foi reiniciado o desmame, com sucesso, sendo retirada a assistência ventilatória mecânica no 57 : $\mathrm{PO}$, passando a nebulizar o tubo $\mathrm{T}$, realizando exercicios respiratórios e exercícios com pressāo positiva com objetivo de recondicionar a musculatura respiratória e manter a expansibilidade pulmonar. A partir desse momento, foi iniciada a deambulação por uma distância de 20 metros, que foi, progressivamente, aumentada de acordo com a tolerância da paciente, sendo que, em uma semana, a distância era de 360 metros. 
COSTA, V. R.; SILVA, A. M. P.; COIMBRA, V. R. M.; FELTRIM, M. I.; JATENE, F. B.; AMATO, M. B. M.; AULER JÚNIOR, J. O. C. - Tratamento alternativo na síndrome da angústia respiratória, no adulto. Rev. Bras. Cir. Cardiovasc., 6(1): 54-58, 1991.

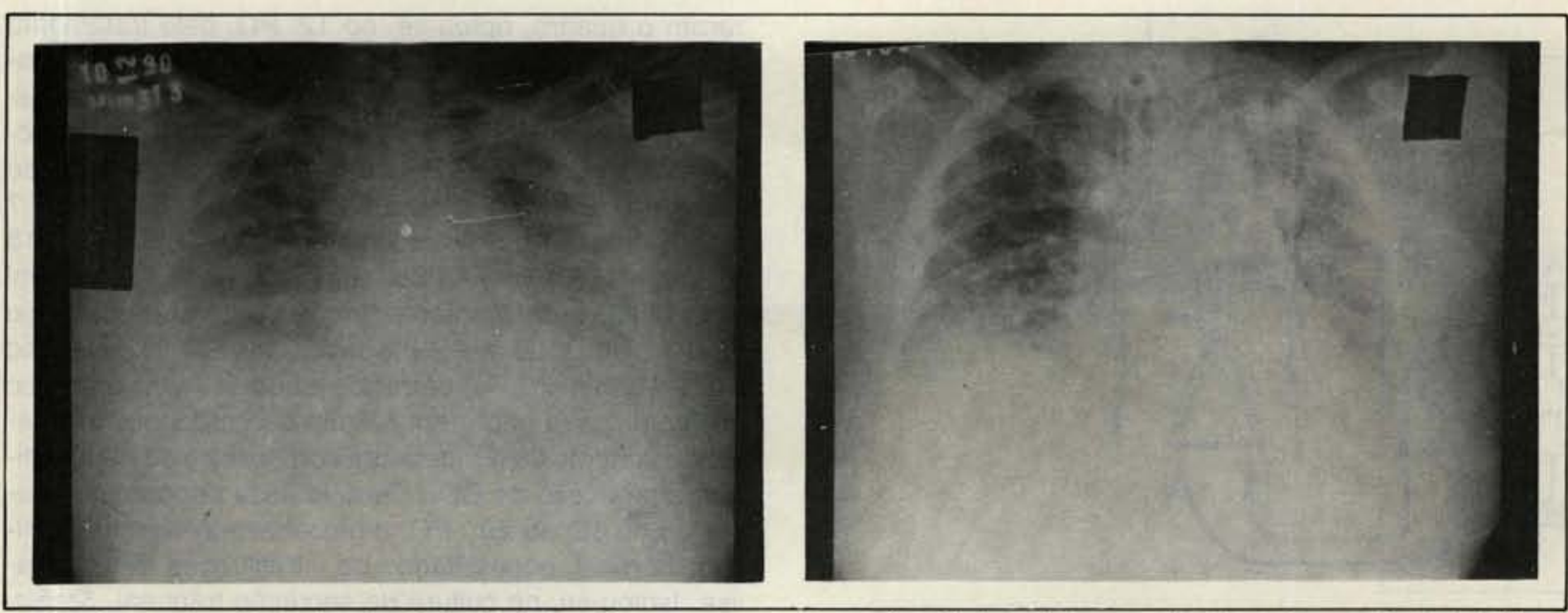

Fig. $2-\mathrm{A}=$ radiografia do tórax do 4: PO pré reinbutação.

$\mathrm{B}=$ radiografia do $30 . \mathrm{PO}$ com a piora do quadro, na primeira tentativa de desmame.

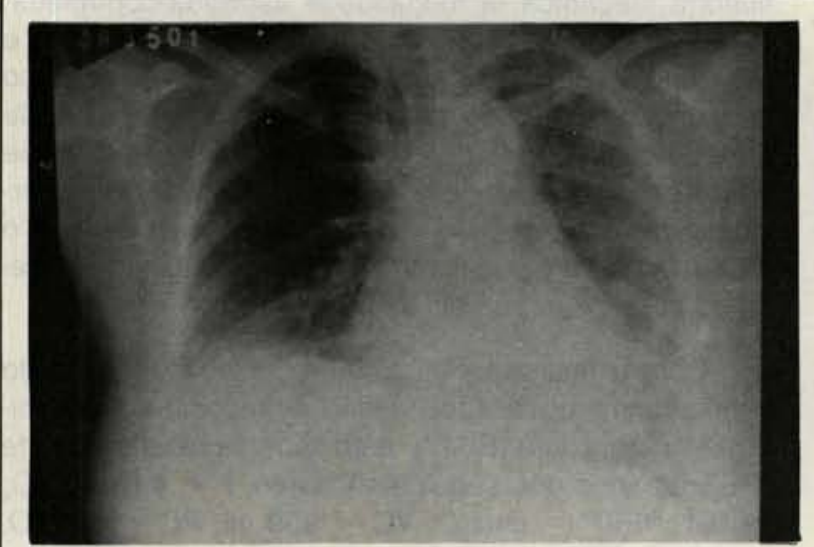

Fig. $3-\mathrm{A}=$ radiografia do tórax da alta hospitalar.

No 68: PO, a paciente recebeu alta da unidade de terapia intensiva, com cânula de traqueostomia metálica $\mathrm{n}$ : 2, fazendo uso de oxigenioterapia. No 72: PO, foi fechado o estoma traqueal e retirado o suporte de $\mathrm{O}_{2}$.

Foram realizadas provas de função pulmonar no 68 . $\mathrm{PO}$, demonstrando insuficiência ventilatória restritiva grau 04 , e no $77^{\circ}$. PO demonstrando insuficiência ventilatória grau 03 .

A alta hospitalar foi no 83: PO (Figura 3A). Quatro meses após, a paciente apresentava RX de tórax norma (Figura 3B). Seis meses após (251: PO), foi realizada nova prova de funçāo pulmonar, cujos resultados demonstraram obstruçāo em pequenas vias aéreas grau I, possivelmente conseqüente à deficiência ventilatória esquerda residual.

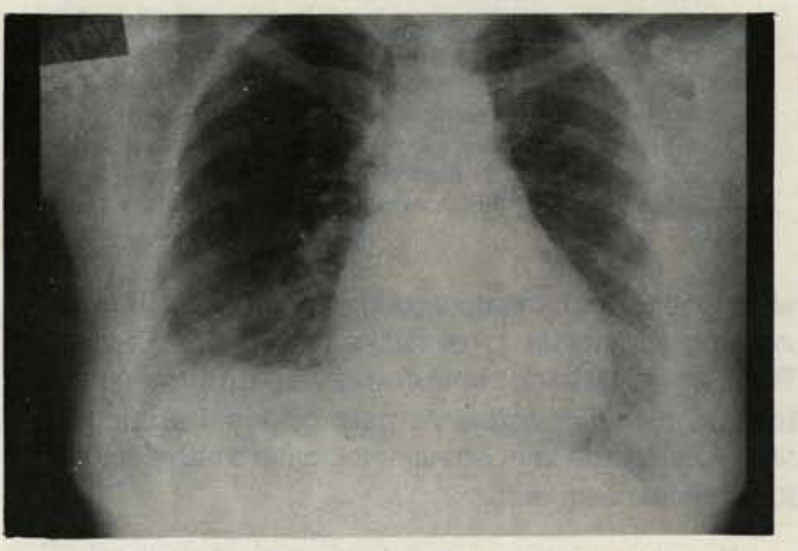

$\mathrm{B}=$ radiografia quatro meses após a alta.

\section{DISCUSSÃO}

A SARA apresenta elevados índices de morbidade e mortalidade, além de difícil tratamento.

O termo SARA foi introduzido, na literatura, há cerca de 24 anos, quando Asbaugh e colaboradores (citados por GATTINONI et alii ${ }^{2}$ ), descreveram, em 12 pacientes adultos, quadro clínico de insuficiência respiratória aguda, caracterizado por dispnéia, hipoxemia e radiografia pulmonar com infiltração difusa e bilateral.

A SARA segue-se a algumas situaçōes clínicas, como estados de choque, infeccções graves, politraumatismos, queimaduras extensas, broncoaspirações e, com menor incidência, intoxicaçāo por diferentes tipos de drogas, inalação de gases tóxicos, circulação extracorpórea, reações anafiláticas graves, pancreatites ${ }^{4,5}$. 
COSTA, V. R.; SILVA, A. M. P.; COIMBRA, V. R. M.; FELTRIM, M. I.; JATENE, F. B.; AMATO, M. B. M.; AULER JÚNIOR, J. O. C. - Tratamento alternativo na síndrome da angústia respiratória, no adulto. Rev. Bras. Cir. Cardiovasc., 6(1): 54-58, 1991.

Clinicamente, a SARA apresenta-se em quatro fases evolutivas ${ }^{1,6}$. A fase inicial é caracterizada por dispnéia e taquipnéia, com radiografia pulmonar normal. $\mathrm{Na}$ segunda fase, acentuam-se os problemas de oxigenação, porém ainda sem mudanças importantes na radiografia. Insuficiência respiratória, hipoxemia acentuada, diminuição de complacência pulmonar e infiltrado difuso na radiografia caracterizam a terceira fase. Finalmente, na quarta fase, a hipoxemia não responde ao oxigênio, intalando-se acidose metabólica. Entretanto, é difícil observar a delimitaçāo exata dessas fases e, freqüentemente, as mesmas confundem-se no tempo. A progressão da insuficiência respiratória depende de muitos fatores, tais como: natureza do agente agressor, fatores concomitantes, como sepsis, grau e extensão da lesão pulmonar e reação do organismo ao tratamento instituído.

$\mathrm{Na}$ SARA observam-se infiltrado de líqüido, perda de pneumócitos I, proliferação septal. O interstício pulmonar é infiltado com células inflamatórias e mesenquimais. Ocorre fibrose pulmonar precoce, obliterando o alvéolo, ductos alveolares e inerstício pulmonar. Os vasos capilares são também afetados e, com freqüência, ocorre falência de múltiplos órgăos, concomitante, ou secundariamente, à SARA ${ }^{1,3,7}$.

Acredita-se que a ativação do sistema complemento induzindo à agregaçăo dos neutrófilos represente importante mecanismo da lesão endotelial desencadeante do edema. A ativação desse sistema é facilmente desencadeada pela endotoxina das bactérias gram-negativas, pelo ácido téććico na sepse por gram-positivos e por outros fatores como as lesōes causadas por queimaduras e exposição de sangue a superfícies não endoteliais, como membranas de dialisadores e oxigenadores ${ }^{1}$. Acredita-se que a lesão endotelial seja mediada por liberação de radicais livres de oxigênio a partir dos neutrófilos ativados que estāo aderidos ao endotélio capilar ${ }^{1}$.

Recentemente, grande interesse tem sido centrado no possível papel dos eicosanóides. Vários metabólitos do ácido araquidônico, gerados por via lipo-oxigenase, podem, virtualmente, mediar os efeitos primários e secundários vistos na SARA. Entre estes, citam-se: hipertensāo pulmonar relacionada às prostaglandinas $A_{2}, B_{2}$, $D_{2}, E_{2}, F_{2}, H_{2}$, e tromboxane ${ }^{1}$; hipoxemia relacionada à prostaglandina $E_{1}(120)$; alterações da mecânica respiratória relacionadas aos leucotrienos $\mathrm{B}_{4}$ e $\mathrm{D}_{4}{ }^{1}$; aumento da permeabilidade mediado pelos leucotrienos $\mathrm{C}_{4}$ e $\mathrm{D}_{4}{ }^{1}$ quimiotaxia dos neutrófilos estimulada pelo leucotrieno $\mathrm{B}_{4}{ }^{1}$.

A irreversibilidade das lesōes pulmonares possui mecanismos ainda nāo bem conhecidos.

Virtualmente, pouco se sabe sobre mensageiros que atuam como intermediários entre o aumento da permeabilidade capilar e a resposta fibrótica que provoca mudanças profundas na mecânica respiratória e na oxigenação sangüínea.
O tratamento da SARA já instalada é basicamente de suporte. Consiste no controle e prevenção da sepse, intensificação do controle hídrico para redução do edema, monitorização das pressões da circulação pulmonar e tratamento da insuficiência respiratória pela ventilação artificial.

A ventilação artificial como coadjuvante do tratamento da SARA progrediu rapidamente. GATTINONI et $a$ ali $^{3}$ demonstraram a melhora clínica e o prognóstico dos pacientes com SARA ventilados artificialmente com pressão positiva ao final da expiração (PEEP).

Outro importante trabalho realizado na década de 70, no "National Heart and Lung Institute", dos Estados Unidos, ordenou estudo prospectivo multicêntrico envolvendo nove instituiçōes norte-americanas, visando ao emprego da circulação extracorpórea em pacientes com SARA, quando métodos convencionais não apresentavam resultados positivos ${ }^{\prime}$. Os resultados obtidos mostraram que a oxigenação extracorpórea năo apresentou benefícios evidentes quando comparada aos métodos convencionais de tratamento. Esse estudo recebeu algumas críticas, no sentido de que grande parte dos pacientes randomizados estavam na fase terminal quando submetidos ao tratamento. Porém esses estudos demonstraram que o suporte extracorpóreo pode ser mais seguro por um período prolongado de tempo, permitindo melhor correção da hipóxia e tempo para recuperação pulmonar.

O termo remoção extracorpórea de $\mathbf{C O}_{2}$ (EC$\left(\mathrm{CO}_{2} \mathrm{O}\right)$ refere-se, principalmente, a acesso veno-venoso com um fluxo sangüíneo entre 20 e $30 \%$ do débito cardíaco. Essa técnica foi desenvolvida em 1979 por GATTINONI et alii ${ }^{3}$ e aperfeiçoada, em 1980 , pelo mesmo grupo, com introdução da técnica de pressão positiva de baixa freqüência associada a $\mathrm{ECCO}_{2} \mathrm{R}$.

Nessa modalide, os pulmōes do paciente são insuflados com PEEP de 15 a $20 \mathrm{cmH}_{2} \mathrm{O}$ e ventilados com f de 3 a 5 rpm com PPI limitada a $35-45 \mathrm{cmH}_{2} \mathrm{O}$, de forma a obter uma distenção intermitente do pulmão.

O suporte pulmonar extracorpóreo consiste, portanto, em uma forma de terapia respiratória que pode substituir a função da troca gasosa, com vantagens de evitar picos de pressão de $\mathrm{FiO}_{2}$ elevados. Os pulmōes são tratados de acordo com as dimensōes atuais do volume pulmonar residual e disponível para ventilação.

A principal complicação da utilização dessa técnica é o sangramento devido ao sistema de coagulação, sendo que a principal causa de morte é a hipoxemia.

A experiência com essa técnica, no Brasil, é recente e reduzida, porém o sucesso obtido com a paciente cujo caso é relatado neste trabalho mostrou ser uma alternativa viável para pacientes com grave comprometimento pulmonar, sem resposta ao suporte ventilatório convencional. 

1991.

Nos dias atuais, vem-se comprovando a necessidade da atuação do fisioterapeuta dentro de unidades de terapia intensiva, pois as novas técnicas de tratamento e o grande número de elevados recursos têm diminuido a mortalidade, levando pacientes a permanecerem por períodos prolongados restritos ao leito e necessitando de assistência ventilatória mecânica.

Tendo em vista a manutenção de tônus e a força muscular, a amplitude articular e a prevenção de escaras, o fisioterapeuta inicia a movimentação dos membros de forma ativa ou passiva, de acordo com o grau de colaboração do paciente, o mais precocemente possível, associado a mudanças de decúbito e à deambulação de forma segura e gradativa.
Nos pacientes sob assistência ventilatória mecânica, o fisioterapeuta trabalha visando manter a permeabilidade das vias aéreas, reeducar a musculatura respiratória e programar o desmame e a retirada da mesma e, também, prevenir complicações e traumatismos traqueais conseqüentes a traqueostomia ou intubação prolongada, controlando a presāo intra-cuff. Para isso, sāo necessárias manobras de higiene brônquica associadas, se necessário, a aspirações endotraqueais, exercícios respiratórios associados à estimulação diafragmática, avaliaçōes constantes da ausculta pumonar, radiografia de tórax, gasometria arterial e ventilometria.

Após a retirada da ventilaçăo mecânica, a fisioterapia tende a enfatizar a terapêutica respiratória, associando exercícios com pressão positiva intermitente, a fim de evitar o retorno do paciente ao respirador.

RBCCV $44205-133$

COSTA, V. R.; SILVA, A. M. P.; COIMBRA, V. R. M.; FELTRIM, M. I.; JATENE, F. B.; AMATO, M. B. M.; AULER JÚNIOR, J. O. C. - Alternative treatment for adult respiratory distress syndrome: case report. Rev. Bras. Cir. Cardiovasc., 6(1): 54-58, 1991.

ABSTRACT: This is a case report of a 33-year-old patient, female, with adult respiratory distress syndrome following coronary by-pass graft surgery. Innitially the patient was supported with mechanical ventilation and PEEP (end expiratory positive pressure) and negative fluid balance. After seven days the $\mathrm{FIO}_{2}$ was $100 \%$ and $\mathrm{PaO}_{2}$ under $50 \mathrm{mmHg}$. A membrane oxygenator device $\left(\mathrm{ECCO}_{2} \mathrm{R}\right)$ was installed to improve arterial oxygenation and $\mathrm{CO}_{2}$ remotion, according to the following protocol: curarization, low tidal volume, and respiratory frequency at the minimal values (under 5 ). After 8 days there was an improvement in arterial oxygenation; the device was removed. The mechanical ventilation was maintained until the 57 th post operative day. After 4 months of follow-up the patient was in good conditions.

DESCRIPTORS: syndrome of respiratory distress, surgery, adults.

\section{REFERÊNCIAS BIBLIOGRÁFICAS}

1 AULER JÚNIOR, J. O. C. - Efeitos de volume e fluxo ventilatórios sobre as propriedades mecânicas do sistema respiratório: estudo em pacientes portadores da síndrome da angústia respiratória do adulto. São Paulo, 1989. [Tese. Livre-Docência. Faculdade de Medicina da Universidade de São Paulo].

2 GATTINONI, L.; PESENTI, A.; CASPANI, M. L. PELIZZOLA, A.; MASCHERONI, D.; MARCOLIN, R.; IAPICHINO, G.; LANGER, M.; AGOSTINI, A.; KOLOBOW, T. The role of static lung compliance in the management of severe ARDS unresponsive to conventional treatment. Intens. Care Med., 10: 121-126, 1984.

3 GATTINONI, P.; PESENTI, A.; MASCHERONI, D.; MARCOLIN, R.; FUMAGALLI, R.; ROSSI, F.; IAPICHINO, G.; ROMAGNOLI, G.; UZIEL, L.; AGOSTINI, A. - Low frequency positive presure ventilation with extracorpo- real $\mathrm{CO}_{2}$ removal in severe acute respiratory falure. JAMA, 256: 881-886, 1986.

4 HICKLING, K. - Extracorporeal $\mathrm{CO}_{2}$ removal in severe adult respiratory distress syndrome. Anaesth. Intens. Care, 14:219-226, 1984.

5 HICKLING, K. - Ventilatory management of ARDS: can it affect the outcome? Intens. Care Med., 16: 219-226, 1984.

6 TOBIN, M. J. \& GRENVIK, A. - Contemporarymanagement in critical care. New York, Churchill Livingstone, 1991.

7 ZAPOL, W. M.; SNIDER, N. T.; HILL, I. D. - Extracorporeal membrane oxigenation in severe acute respiratory failure: a randomised prospective study. JAMA, 242: 2193-2196, 1979. 